\title{
Optimization of Stirling engine operation based on mathematical modeling
}

\author{
Aleksandr Makarovich Gaponenko \\ Department of Heat Power Engineering and Heat \\ Engineering \\ Kuban State Technological University \\ Krasnodar, Russia \\ gaponenko.am@yandex.ru
}

\author{
Aleksandra Aleksandrovna Kagramanova \\ Department of Heat Power Engineering and Heat \\ Engineering \\ Kuban State Technological University \\ Krasnodar, Russia \\ kaa_200110@mail.ru
}

\begin{abstract}
The opportunity of application of Stirling engine with non-conventional and renewable sources of energy was considered in the paper. The advantage of such use was demonstrated. The expression for the thermal efficiency of the Stirling engine was obtained. It was shown that the work per cycle is proportional to the amount of substance, and, hence, to the pressure of the working fluid, to the temperature difference. The work per cycle, to a lesser extent, depends on the expansion coefficient. The efficiency of ideal Stirling cycle coincides with the efficiency of an ideal engine, working according to the Carnot cycle, which compares favourably a Stirling cycle with the cycles of Otto and Diesel underlying the engine. It has been established that among the four input parameters, there is only one parameter which can be easily changed during operation and which influences effectively the operation of the engine. This parameter is the phase difference. The paper presents results of optimization of various temperature parameters, corresponding to high and low potential heat sources for various values of the ratio of the compression chamber to the volume of the expansion chamber. The optimal value of this parameter depends on the conditions under which the engine is designed. The mathematical model of Schmidt cycle was considered, and the operation of Stirling engine in the approximation of Schmidt by means of numerical analysis is analysed. To conduct numerical experiments, the program-function in the MathLab language was developed. The results of numerical experiments are illustrated by graphical charts.

Keywords-optimization, cluster approach, theory of random processes, simulation model of hybrid cluster
\end{abstract}

\section{INTRODUCTION}

\section{A. Overview of possible applications and advantages of the Stirling engine}

The objects of exploration, development of oil and gas wells, oil and gas extraction are often located in remote, inaccessible places, far from electrical networks and highways. Diesel power plants currently supply autonomous power of these objects, which is connected with necessary supply of fuel and associated large overhead expenses. In this regard, the use of non-traditional renewable sources of energy (NRSE) is of particular relevance.

The following renewable sources of energy are well known: wind energy; geothermal heat; solar radiation absorbed by either using solar panels in the form of electricity or converted into thermal energy through solar collectors. This group should also include local energy sources such as energy of associated gas, peat, firewood, coal, coal shale, etc. Because of their specificity to ensure reliable energy, these sources must be integrated in the local energy sector, which should provide the necessary heat for heating dwellings and places of staff residence, and electricity needed for appliances and installations. Previous studies of the authors have shown the importance of optimal composition of the energy complex, based on RES. As part of the local energy, this mix should include a converter of thermal energy into electrical energy, which has a number of properties: versatility of consumed energy, great lifespan, absence of necessity in regular maintenance, reliability, low noise, high power density. The only currently known transducer of this type is the Stirling generator, which represents a heat engine in which the generator is combined with a Stirling engine.

The Stirling engine (DS) is referred to internal combustion engines, more specifically to the engine with external supply of heat. For operating DS, it is necessary to provide the temperature difference in its heat exchangers. The heater can use the heat of the burner, steam or water, heated in the geothermal or solar collectors. It is possible to directly supply the solar energy from the solar concentrator. For these cases, a special design of DS with a quartz head, through which concentrated solar radiation enters directly into the expansion chamber, is provided. For these engines, it is possible to use even geothermal water, pumped directly from the well, or associated oil gas, which can have high corrosion aggressiveness. It is possible to use ambient medium, chilled water, ice or snow as a refrigereator.

The peculiarity of the Stirling engine is that it operates in a closed cycle, which allows one to fully seal the body itself and to apply helium or hydrogen used as a working fluid. This 
ensures high pressure of the working fluid, which increases the power density (power per unit volume or mass of the engine). In the Stirling Engine, there is no combustion chamber, valve, a power supply system, a fuel system. In some types of Stirling engine, such as the free-propeller Beal engine, there is no crank mechanism and no crankshaft; there is no transmission, which transfers traffic from one node to another. The latter allows one to completely abandon the lubricants, using special materials as seals. In the operation of the engine, there are no explosions, no burned exhaust gas characteristic of the engine. These structural features lead to exceptionally low noise and unprecedented service life, reaching tens or hundreds of thousands of hours of continuous operation with no maintenance.

In Stirling engines, there is no trigger since the engine has the property of self-starting, according to which it begins to work during heat supply and stops in case of its termination. This property simplifies the use of Stirling engines, especially in remote, hard-to-reach areas, for example on the mast of solar concentrators.

The desire to use all the advantages of the Stirling engine induced numerous studies, conducted by research centers of various firms, primarily by the firm "Philips" since the mid 30s, and then, under licence, by "Phillips", "General motors", "Man-MVM", "United Stirling", etc. As a result of these studies, designs in a wide range of capacities - from a few watts, to charge the batteries, up to several thousand kilowatts, intended for the engines of buses, heavy vehicles, ships, and submarines - were developed. However, nowadays these engines have not been widely used, remaining often the subject of research and development due to both economic (high cost of development, competition) and commercial (patent limitations, the preservation of trade secrets) reasons. Therefore, in public media, there are almost no research results and descriptions of the development of modern engines despite the popularity of the topic.

Despite these circumstances, DS is promising, and it is necessary to work on it right now. In order to place orders for the development, debugging, acquisition of necessary models, it is necessary at least to imagine the influence of certain design parameters on the performance of the engines. For this, it is necessary to have a simple but fairly appropriate mathematical model.

\section{B. Principle of Stirling engineoperation. Mathematical models}

The Stirling engine [1-5] works in a closed cycle, according to which the working fluid expands at higher temperatures and contracts at much lower temperature, which ensures gains in its operation.

A prototype of the real cycle is the ideal Stirling cycle, consisting of two isotherms and two isochores. A Stirling machine can operate in the direct cycle (in this case, it is an engine) as well as in the reversible cycle (in this case, it is a refrigeration machine). The expansion of the working fluid occurs at a higher temperature of heater $T_{1}$ and contraction at refrigerator temperature $T_{2}$ of isothermal processes. The transition of the working fluid between these temperatures is carried out during the two isochoric processes.

Let us obtain an expression for cycle efficiency. The working fluid receives heat during isochoric heating and isothermal expansion:

$$
Q_{H}=q_{1}+Q_{1}=v \cdot C_{V} \cdot\left(T_{1}-T_{2}\right)+v \cdot R \cdot T_{1} \cdot \ln \frac{V_{2}}{V_{1}},(1)
$$

and gives it up during isochoric cooling and isothermal compression:

$$
Q_{x}=q_{2}+Q_{2}=v \cdot C_{V} \cdot\left(T_{1}-T_{2}\right)+v \cdot R \cdot T_{2} \cdot \ln \frac{V_{2}}{V_{1}}
$$

where:

$\mathrm{Q}_{1}$ - the amount of heat, received by working fluid during isothermal expansion;

$\mathrm{q}_{1}$ - the amount of heat, received by working fluid during isochoric heating;

$\mathrm{Q}_{2}$ - the amount of heat, received by the working fluid during isothermal compression;

$\mathrm{q}_{2}$ - the amount of heat, received by working fluid during isochoric cooling;

$v$ is the number of moles of the substance, $\mathrm{C}_{\mathrm{V}}$ is the molar heat capacity with constant volume.

The work, performed per cycle is equal to:

$$
A=Q_{1}-Q_{2}=v \cdot R \cdot \ln \frac{V_{2}}{V_{1}} \cdot\left(T_{1}-T_{2}\right)
$$

The coefficient of thermal efficiency is:

$$
\eta=\frac{A}{Q_{H}}=\frac{v \cdot R \cdot \ln \frac{V_{2}}{V_{1}} \cdot\left(T_{1}-T_{2}\right)}{v \cdot R \cdot T_{2} \cdot \ln \frac{V_{2}}{V_{1}} \cdot T_{1}+q_{1}}
$$

Stirling proposed obtaining heat $\mathrm{q}_{1}$ not from the heater, but from the regenerator, into which the heat enters as a result of heat exchange during isochoric cooling of the gas. In this case, $\mathrm{q}_{1}$ disappears from the denominator and formula (4) has the form:

$$
\eta=\frac{T_{1}-T_{2}}{T_{1}}
$$

i.e., it coincides with the efficiency of a perfect engine. From this, one can draw the following important conclusions:

The work per cycle is proportional to the amount of substance, and hence to the pressure of the working fluid, to the temperature difference, and, to a lesser extent, depends on the expansion coefficient $\mathrm{V}_{2} / \mathrm{V}_{1}$.

The efficiency of the ideal Stirling cycle coincides with the efficiency of an ideal engine, operating by the Carnot cycle, which compares a Stirling cycle favourably with the cycles of Otto and Diesel, underlying the engine.

Schmidt, using the representation about harmonic motion of pistons and the rest of the nodes, has left other assumptions unchanged [6]. They are the equality of the instantaneous pressure at any point in the volume; ideal regeneration; isothermality of expansion and contraction; the instantaneous 
change of the temperature of gas, entering the corresponding cell (perfect heat transfer); the ideal gas used as the working fluid. Thus, the scheme of Schmidt, although being approximate, is undoubtedly more realistic than the ideal Stirling cycle.

Let us turn to the derivation of the mathematical model of Schmidt cycle.

Let us assume that the working fluid is a perfect gas, obeying Mendeleev-Clapeyron equation $P \cdot V=V \cdot R \cdot T$, whence an expression for the number of moles of substance $v=\frac{P \cdot V}{R \cdot T}$ is obtained. Taking into account that the entire gas is distributed into three volumes: expansion volume $\mathrm{V}_{\mathrm{E}}$, being at temperature $\mathrm{T}_{1}$; compression volume $\mathrm{Vc}$, being at temperature $\mathrm{T}_{2}$; and dead volume $\mathrm{V}_{\mathrm{D}}$ - at temperature $T_{D}=\frac{T_{1}+T_{2}}{2}$, let us calculate the total amount of substance:

$$
v=v_{1}+v_{2}+v_{3}=\frac{P}{R} \cdot\left(\frac{V_{E}}{T_{1}}+\frac{V_{c}}{T_{2}}+\frac{V_{D}}{T_{D}}\right),(6)
$$

whence the expression for the pressure is obtained:

$$
P=\frac{v \cdot R}{\frac{V_{E}}{T_{1}}+\frac{V_{c}}{T_{2}}+\frac{V_{D}}{T_{D}}} .
$$

In accordance with Schmidt's assumption about harmonic motion, let us present the dependence of the expansion volume and contraction volume on the angle of shaft rotation, calculated relatively the top dead center of the expansion piston:

$$
V_{E}=\frac{V_{0}}{2} \cdot(1+\cos \varphi) ; V_{c}=\frac{k \cdot V_{0}}{2} \cdot(1+\cos (\varphi-\delta)),
$$

where $\mathrm{V}_{0}$ - the amplitude of volume $\mathrm{V}_{\mathrm{E}}$;

$\varphi$ - the rotation angle of the crankshaft (process phase);

$\delta$ - the lag by the phase of the compression piston from the expansion piston.

Let us also suppose that $\tau=\frac{T_{1}}{T_{2}} ; \quad V_{D}=X \cdot V_{0}$; $T_{D}=\frac{1}{2} \cdot\left(T_{1}+T_{2}\right)$. The expression for pressure takes the following form:

$$
P=\frac{v \cdot R \cdot T_{2}}{V_{0}} \cdot \frac{2}{\frac{1}{\tau} \cdot \cos \varphi+k \cdot \cos (\varphi-\delta)+\frac{2 x}{(1+\tau)}} .
$$

The work during gas expansion is equal to:

$$
A_{E}=\oint P_{E} \cdot d \cdot V_{E}=-v \cdot R \cdot T_{2} \oint \frac{\sin \varphi \cdot d \cdot \varphi}{\frac{1}{\tau} \cdot \cos \varphi+k \cdot \cos (\varphi-\delta)+\frac{2 x}{(1+\tau)}} .
$$

The work during compression is:

$$
A_{C}=\oint P_{c} \cdot d \cdot V_{c}=-v \cdot R \cdot T_{2} \oint \frac{k \cdot \sin \varphi \cdot d \cdot \varphi}{\left.\frac{1}{\tau} \cdot \cos \varphi+k \cdot \operatorname{co} \$ \varphi-\delta\right)+\frac{2 x}{(1+\tau)}} .
$$

Here, symbol $\oint$ indicates integration by cycle: $\oint=\int_{\varphi=0}^{2 \pi}$ The total work per cycle is equal to:

$$
A=A_{E}+A_{c} \text {. }
$$

When performing the numerical integration in the abovementioned formulas, it is possible to make up the function that allows one to obtain numerically the value of the work per cycle, according to the set of input parameters. Such function was created in the MATHLAB language and used to analyze the operation of the Stirling engine in the approximation of Schmidt.

\section{COMPUTATIONAL EXPERIMENTS OF THE STIRLING ENGINE OPERATION IN THE APPROXIMATION OF SCHMIDT AND THEIR ANALYSIS}

The purpose of the analysis is to identify the dependence of the dimensionless parameters, characterizing the performance of cycle $\left(\frac{P}{P_{0}}, \frac{V_{1}}{V_{0}}, \frac{V_{2}}{V_{0}}, \frac{A}{v R T_{0}}, \frac{Q_{1}}{v R T_{0}}, \frac{Q_{2}}{v R T_{0}}\right)$, on the dimensionless parameters, characterizing the design features of the process, its mode or condition $\tau, k, X, \delta$. For conducting numerical analysis, a program-function in the MATHLAB language was drawn up. Using this program, it is possible to analyze the DS work to reveal the influence of various design parameters on its performance and to determine the appropriate combination of parameters, optimizing the engine performance per cycle, as it will be shown below.

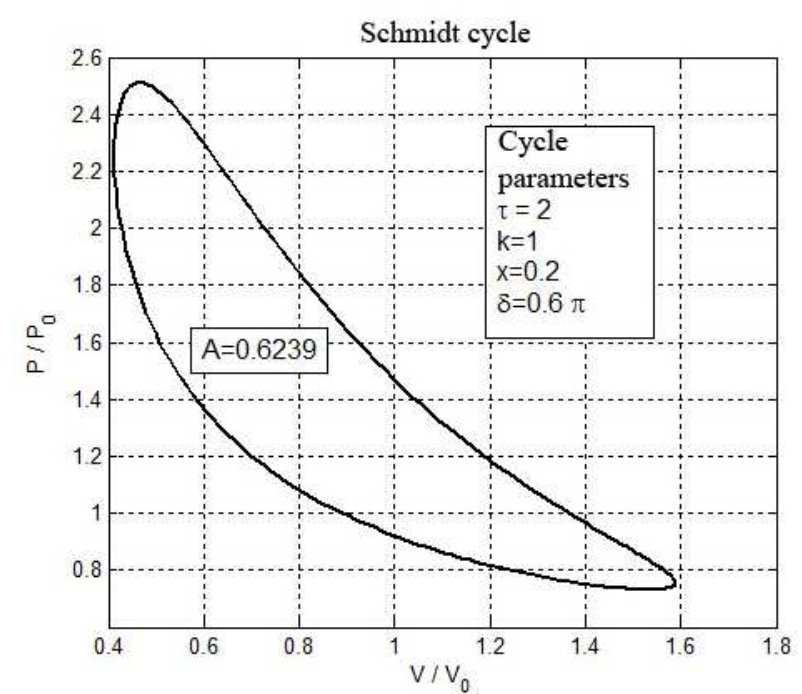

Fig.1. Cycle form and value of dimensionless operation with certain values of parameters

The calculations showed that during perfect operation of the regenerator, the efficiency of the Schmidt cycle coincides with the efficiency of DS or that of the Carnot cycle.

Dimensionless operation per cycle $\frac{A}{v \cdot R \cdot T_{2}}$ depends on four dimensionless parameters: $\delta, \tau, \mathrm{k}, \mathrm{X}[7-10]$. Phase difference $\delta$ 
exerts especially noticeable influence so that its value can control the output of a heat engine: if $0<\delta<\pi, A>0$, the system operates as an engine; if $\pi<\delta<2 \pi, A<0$ - as a refrigerating machine. Let us call the dependence of the dimensionless operation on the phase difference as a phase characteristic. The maximum value of operation (value $\delta$ ), under which the maximum takes place, as well as the kind of the phase characteristic, depends on the values of the three remaining parameters. Therefore, to characterize the influence of these parameters, it is convenient to represent the family of phase curves in the graph, depending on any of the three parameters at constant values of other two parameters. The following graph shows the family of phase characteristics under various values of parameter $\mathrm{k}$.

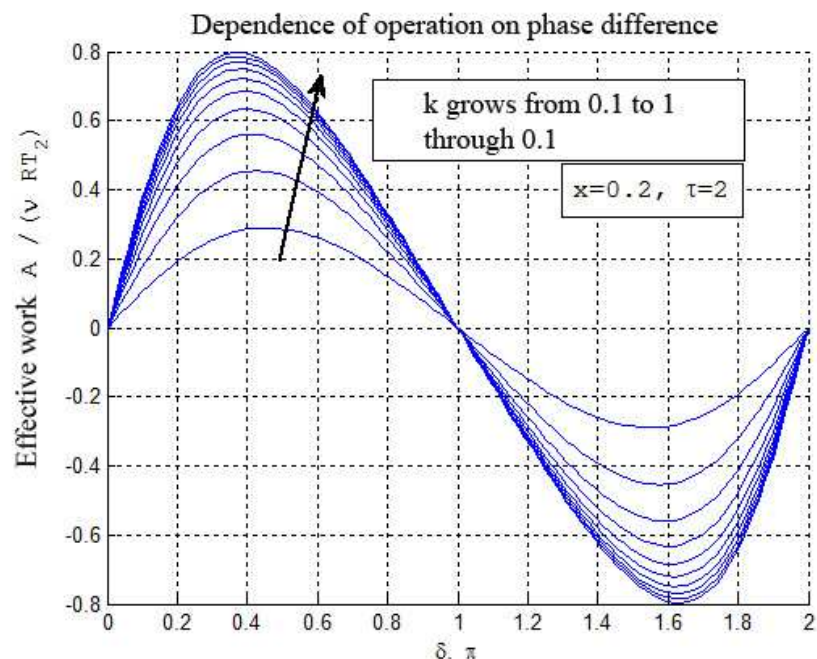

Fig. 2. Dependence of phase characteristic on ratio of volumes $k=V c / V_{E}$ when $k \leq 1$. The arrow shows the sequence of change of the curve in the family with the specified change of corresponding parameter $\mathrm{k}$

The graph shows the influence of phase difference $\delta$ on the value of the work per cycle. Taking into consideration that value $\delta$ is technically easy to change in the process of engine operation, the indicated parameter can be used for power control of Stirling engines.

Many designs of Stirling engines vary by the ratio of volumes of expansion and contraction chambers $k=V_{c 0} / V_{0}$.

From the previous picture, one may see that value $\delta=\delta_{m}$, maximizing value $\mathrm{A}$, is different for different values of $\mathrm{k}$. This value, as well as value $\mathrm{A}_{\mathrm{opt}}$, depends on two other parameters: $\tau=\frac{T_{1}}{T_{2}}$ and $X=\frac{V_{D}}{V_{0}}$.

Given that the value of $\delta$ can be adjusted while in service, whereas $\mathrm{k}$ and $\mathrm{X}$ are rigidly connected with the engine design, and the value of $\tau$, associated with operating temperature, can also be considered constant. The optimization by $\mathrm{k}$ can be realized as follows: by setting specific values of $\tau$ and $X$, by optimizing $\mathrm{A}$ for each value of $\mathrm{k}$ from a fairly wide range in the neighborhood of the optimum, having identified $\mathrm{A}_{\mathrm{m}}(\mathrm{k})$ and $\delta_{\mathrm{m}}(\mathrm{k})$, and then, by determining optimum $\mathrm{k}$ from the obtained values.

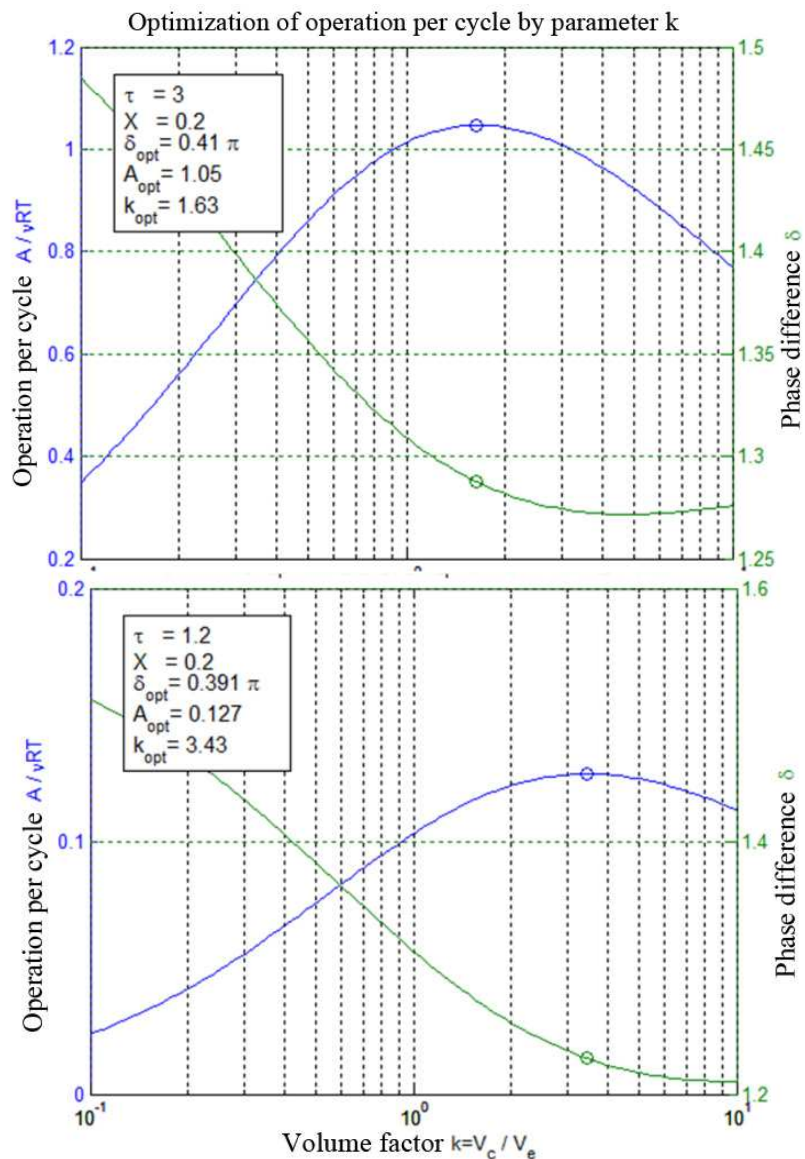

Fig.3. Optimization of Stirling engine by volume factor $\mathrm{k}$ for different values of $\tau$ with constant value $\mathrm{X}$. Curves show $\mathrm{A}_{\mathrm{m}}(\mathrm{k})$ (in dimensionless form) and $\delta_{\mathrm{m}}(\mathrm{k})$. Round markers indicate $A_{\text {opt }}\left(k_{\text {opt }}\right)$ and $\delta_{\text {opt }}\left(k_{\text {opt }}\right)$

The result of the MATHLAB program operation, which uses the previously described function to perform such optimization and output in a graphic window of diagrams $\mathrm{A}_{\mathrm{m}}(\mathrm{k}), \delta_{\mathrm{m}}(\mathrm{k})$ of values $\mathrm{A}_{\mathrm{opt}}, \delta_{\mathrm{opt}}$ and the corresponding annotation, is given below.

It follows from the figure that the optimum value of $\mathrm{k}$ should be selected in advance, based on the planned temperature range of the engine. Thus, $\tau=3$, which corresponds to $T_{1}=900$ $\mathrm{K}\left(627^{\circ} \mathrm{C}\right), \mathrm{T}_{2}=300 \mathrm{~K}\left(27^{\circ} \mathrm{C}\right), \mathrm{k}_{\mathrm{opt}}=1,63$; whereas $\tau=1,2$, which corresponds to heater temperature $\mathrm{T}_{1}=360 \mathrm{~K}\left(87^{\circ} \mathrm{C}\right)$ at the same temperature of the refrigerator. The latter corresponds to using low-grade heat of hydrothermal reservoirs.

Each Stirling engine has dead volume $V_{D}$, which includes the volume of the heater, the regenerator, the refrigerator and other ballast areas, where the working fluid is not involved in the processes of compression-expansion. When designing a Stirling engine, it is important to have an idea about how the proportion of dead volume influences the engine operation. The following two pictures, drawn on the basis of numerical calculations, explain this influence. 


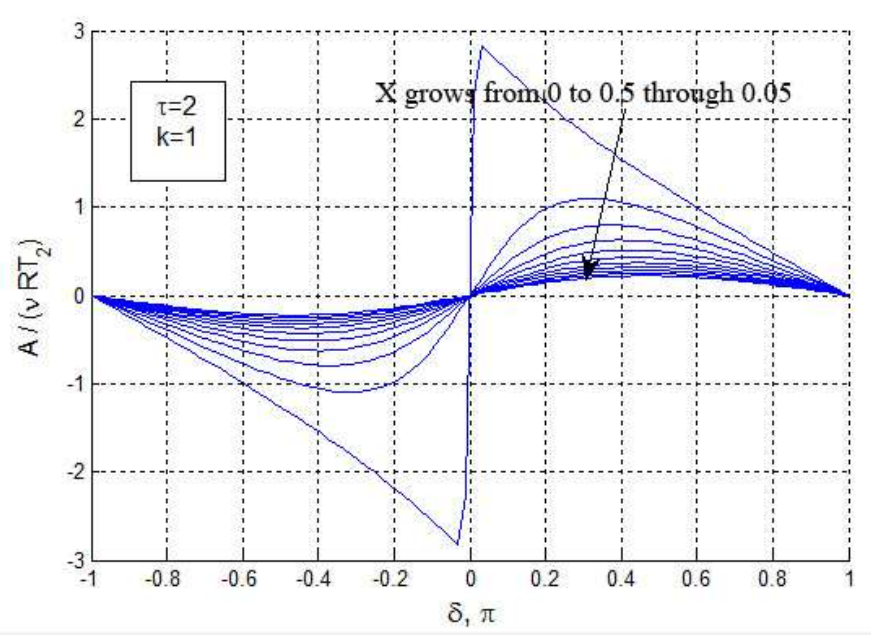

Fig.4. Dependence of phase characteristic on proportion of dead volume

The figure shows that with the increase of dead volume, the optimal phase shift varies almost from 0 to $\frac{\pi}{2}$, and with zero volume, the maximum point is unstable. With a small spontaneous decrease of $\delta$, the process can move from a positive to negative (the engine mode switches to the mode of refrigerating machine). From this, it is possible to deduce that, although with the growth of the dead volume proportion the maximum work decreases, some of its amount is necessary for the stable DS operation.

Figure 5 shows in more detail the dependence of operation, optimized by $\delta$, and optimizing phase difference on the proportion of dead volume X.

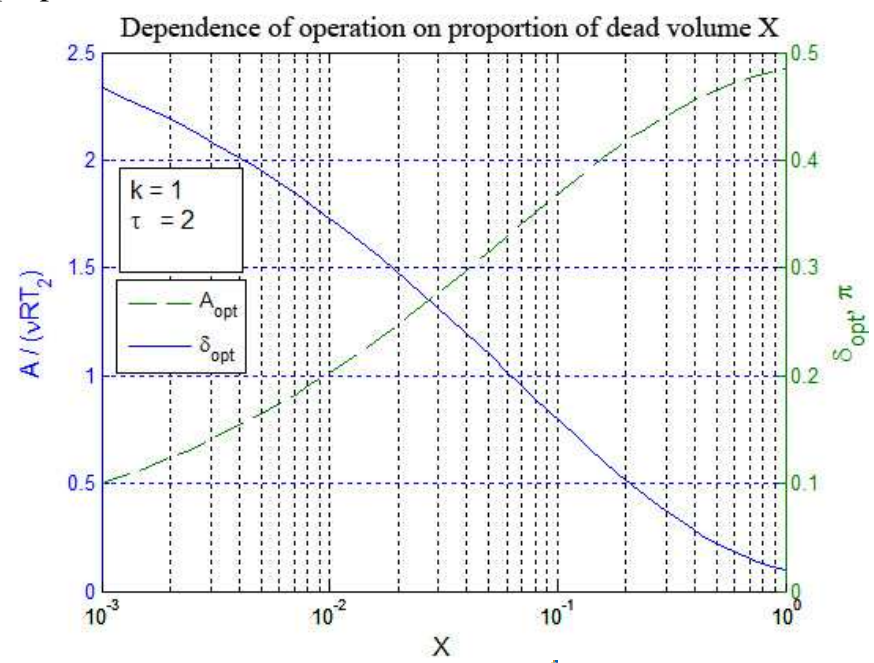

Fig.5. Dependence of dimensionless operation $\frac{A}{w R T_{2}}$, optimized by $\delta$ on the proportion of dead volume $\mathrm{X}$ and corresponding optimizing values $\delta_{\text {opt }}$

Figure 5 shows that the growth of the dead volume proportion significantly influence the engine performance degradation.

When using DS with various sources of thermal energy, it is important to know how the temperature difference of the heater and the refrigerator influences the engine performance. In a dimensionless form, the temperature difference relatively ambient temperature (refrigerator) is expressed by parameter $\tau$. To obtain the dependence of operation on the temperature difference, an approach, similar to that used to obtain the graphs of Fig. 5, was applied. For each $\tau$ from a certain range, value A was optimized by $\delta$, and obtained points $\left(A_{o p t}, \tau\right)$ and $\left(\delta_{o p t}, \tau\right)$ were displayed in the form of curves on the diagram. The dependence of the engine efficiency on the temperature difference in the dimensionless form is shown in Figure 6.

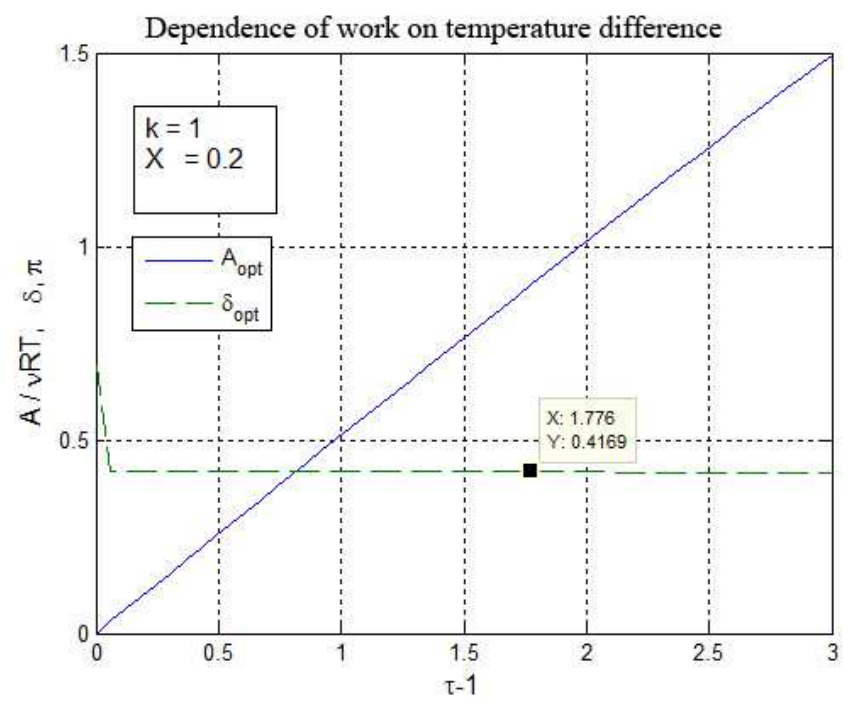

Fig.6. Dependence of operation per cycle on temperature difference

Figure 6 shows that for preset $\mathrm{k}$ and $\mathrm{X}$, the work grows almost proportionally to the temperature difference with almost constant optimal $\delta$.

\section{CONCLUSIONS:}

The structure of the local energy complex should include a converter of thermal energy into electrical energy - Stirling generator - a compact generator, combined with the Stirling engine (DS), which have a number of properties. They are versatility of consumed energy, great lifespan, absence of necessity of regular maintenance, reliability, low noise, high power density.

It is shown that with perfect regeneration and without heat losses, the DS efficiency coincides with the efficiency of a Carnot cycle, which is the maximum possible efficiency.

The authors demonstrated that the operation per cycle is proportional to the amount of substance of the working fluid, and thereby to its pressure at ambient temperature.

The paper presents the mathematical model of the Schmidt cycle, on the basis of which the program, written in MATHLAB, is composed, allowing one to analyze the DS operation by four input parameters to determine a suitable combination of design parameters and their influence on the efficiency of the engine operation. The results of such research are given.

It was established that among four input parameters, the only parameter that can be easily changed during operation and which effectively influences the engine operation is phase difference $\delta$. The dependence of the operation per cycle on $\delta$, 
called in the paper as a phase characteristic, illustrates clearly the mode of DS operation. The families of phase characteristics for different operation modes are presented.

The paper presents the results of optimization for different temperature parameters, corresponding to high and low potential sources of heat for various values of the ratio of the volumes of the compression chamber to the expansion chamber (k). The optimal value of this parameter depends on the conditions, for which the engine is meant.

The results of the corresponding calculations, from which it is concluded that the decrease in the proportion of dead volume (X) leads to a significant increase in operation efficiency, are given. However, extremely small dead volume can lead to a loss of stability of engine operation.

It is shown that operation $\mathrm{X}$ per cycle, being the maximum by $\delta$, with constant values of $\mathrm{k}$ is almost proportional to the temperature difference between the heater and the fridge.

\section{References:}

[1] G.V. Smirnov, External combustion engine, "Knowledge", M., 1967.

[2] G. Walker, Machine operating on the Stirling cycle, M.: Energy, 1978, p. 152.

[3] G. Walker, Stirling Engines, M.: Mashinostroenie, 1985, p. 408.

[4] M. G. Kruglov, The Stirling Engines, M.: Mashinostroenie, 1977, p. 150 .

[5] G. Reader, P. Hooper, Stirling Engines, M.: Mir, 1986, p. 464.

[6] K. V. Frolov, Mechanical engineering. The encyclopedia in forty volumes (24 volumes), M.: mechanical engineering, 2010, p. 458.

[7] A.M. Gaponenko, A.A. Kagramanova, "Methodology and algorithm of mathematical analysis multifactor models optimal configuration of renewable energy sources,” International research journal, №4(46), Vol. II, April 2016, pp. 76-79.

[8] A.M. Gaponenko, A.A. Kagramanova, "Method of determination of optimal parameters of energy efficient of power supply systems," International research journal, №4 (46), Vol. II, pp. 80-84, April 2016.

[9] I. E. Anufriev, A. B. Smirnov, E. N. Smirnova, MATLAB 7, SPb.: BHV-Petersburg, 2005, p. 1104.

[10] D. R. Bren, Theory of linear viscoelasticity, M.: Mir, 1965, p. 390. 